\begin{tabular}{|c|c|}
\hline Title & Magnetic Field and Fluid Flow Computation of Plural Kinds of Magnetic Particles for Magnetic Separation \\
\hline Author(s) & Noguchi, So; Kim, SeokBeom \\
\hline Citation & $\begin{array}{l}\text { IEEE Transactions on Magnetics, 48(2), 523-526 } \\
\text { https://doi.org/10.1109/ MA G.2011.2174207 }\end{array}$ \\
\hline Issue Date & $2012-02$ \\
\hline Doc URL & http:/hdl.handle.net/2115/48512 \\
\hline Rights & $\begin{array}{l}\text { (9) } 2012 \text { IEEE. Reprinted, with permission, from So Noguchi, SeokBeom Kim, Magnetic Field and Fluid Flow } \\
\text { Computation of Plural Kinds of Magnetic Particles for Magnetic Separation, IIEEE Transactions on Magnetics, Feb. } \\
2012 \text {. This material is posted here with permission of the IEEE. Such permission of the IEEE does not in any way imply } \\
\text { IEEE endorsement of any of Hokkaido University products or services. Internal or personal use of this material is } \\
\text { permitted. However, permission to reprint /republish this material for advertising or promotional purposes or for creating } \\
\text { new collective works for resale or redistribution must be obtained from the IEEE by writing to pubs } \\
\text { permissions@ ieee.org. By choosing to view this document, you agree to all provisions of the copyright laws protecting } \\
\text { it. }\end{array}$ \\
\hline Type & article (author version) \\
\hline File Information & ToM48-2_523-526.pdf \\
\hline
\end{tabular}

Instructions for use 


\title{
Magnetic Field and Fluid Flow Computation of Plural Kinds of Magnetic Particles for Magnetic Separation
}

\author{
So Noguchi ${ }^{1}$ and SeokBeom Kim ${ }^{2}$ \\ ${ }^{1}$ Graduate School of Information Science and Technology, Hokkaido University, Sapporo 060-0814, Japan \\ ${ }^{2}$ Graduate School of Natural Science and Technology, Okayama University, Okayama 700-8530, Japan
}

\begin{abstract}
Magnetic chromatography is an effective system for fine magnetic particle separation because of its strong magnetic field gradients. We have been developing the magnetic chromatography system to separate two or more kinds of fine magnetic particle with different sizes. To evaluate the performances of our developed system, we have also developed a simulation code with taking into account the fluid dynamics and magnetics. It is, however, difficult to consider two or more kinds of fine magnetic particles with different sizes in the simulation, because magnetic interferences between two or more kinds of magnetic particles need to be considered. Therefore, we have newly developed a simulation code to deal with magnetic interferences between two or more kinds of fine magnetic particles.
\end{abstract}

Index Terms-Magnetic chromatography, magnetic fluid, magnetic particle separation, numerical analysis.

\section{INTRODUCTION}

M AGNETIC SEPARATION is a useful system for separating fine magnetic particles because of its strong magnetic field gradients in a very small flow channel. Therefore, various magnetic separation systems have been designed, developed and demonstrated [1]-[4]. We have also developed a magnetic chromatography system in order to separate the fine magnetic particles and ions [5]-[7]. In a developed magnetic chromatography system, ferromagnetic wires width of $200 \mu \mathrm{m}$, that generate the magnetic field gradients, are located on the upper and lower walls. We had expected that the large particles with a large magnetic susceptibility were extracted in a radial direction of the flow channel and concentrated around the channel wall, and the particles with a small magnetic susceptibility could pass through the channel with weak attraction to the channel wall. In order to confirm the magnetic force attracting for the wall and flow of the fine magnetic particles, we have developed a simulation code coupling the fluid dynamics and magnetics [8].

Some simulation results coupled electromagnetics and fluid dynamics have been reported for various applications [9]-[12]. Some designs of magnetic separation were performed based on a calculation of the magnetic field gradients, not considering the fluid flow [1]. The fluid flow was simulated based on the 2D fluid dynamics analysis coupling with a simplified magnetic field analysis [6], [7]. The simplified magnetic field analysis was conducted only once as a precondition, though the time-varying concentration of magnetic particles affects the magnetic field and its gradients. Hence, to accurately simulate the magnetic fluid flow for magnetic separation, we have developed the $3 \mathrm{D}$ simulation code taking into account the Navier-Stokes, control volume, and magnetic field equations simultaneously [8]. The NavierStokes equation can represent a fluid flow. The concentration of magnetic particles considering its diffusion is represented by the control volume equation. The magnetic field has to be

Manuscript received July 7, 2011. Corresponding author: S. Noguchi (email: noguchi@ssi.ist.hokudai.ac.jp).

Digital Object Identifier inserted by IEEE solved since its gradients changes as a concentration of the magnetic particles changes. We have confirmed that the developed simulation results adequately agreed with the experimental results [8].

We could not obtain the magnetic separation against the magnetic particles which have different sizes by previously developed simulation code, since it was capable handle with only one kind of magnetic particle. To deal with two or more kinds of magnetic particle with different sizes the volume control and the magnetic field simulations are modified.

\section{NUMERICAL ANALYSIS FOR Two KindS OF PARTICLES}

\section{A. Magnetic Field Analysis}

The magnetic fluid would receive magnetic forces based on magnetic field gradients. Therefore, in the simulation to study the behaviors of the magnetic fluid, the magnetic field characteristics with high accuracy have to be considered. However, a scale of magnet is much larger than that of magnetic fluid, because a superconducting magnet, whose scale is large enough, is usually employed to generate a high magnetic field. In this study, the magnetic moment method is employed since the integral method is more accurate than the domain method, such as the finite element method.

Using the magnet moment method [13], the magnetization $\boldsymbol{M}$ is obtained from

$$
\begin{aligned}
& -\frac{1}{4 \pi} \int_{V}\left(\nabla^{\prime} \cdot \boldsymbol{M}\left(\boldsymbol{r}^{\prime}\right)\right) \frac{\boldsymbol{r}-\boldsymbol{r}^{\prime}}{\left|\boldsymbol{r}-\boldsymbol{r}^{\prime}\right|^{3}} \mathrm{~d} V^{\prime}+ \\
& \frac{1}{4 \pi} \int_{S}\left(\boldsymbol{M}\left(\boldsymbol{r}^{\prime}\right) \cdot \boldsymbol{n}\right) \frac{\boldsymbol{r}-\boldsymbol{r}^{\prime}}{\left|\boldsymbol{r}-\boldsymbol{r}^{\prime}\right|^{3}} \mathrm{~d} S^{\prime}-\frac{1}{\chi_{\mathrm{m}}(\boldsymbol{r})} \boldsymbol{M}(\boldsymbol{r})=-\boldsymbol{B}_{\mathrm{F}}(\boldsymbol{r})
\end{aligned}
$$

where $\boldsymbol{r}$ and $\boldsymbol{r}^{\prime}$ are the position vector, $\chi_{\mathrm{m}}$ the susceptibility, and $\boldsymbol{B}_{\mathrm{F}}$ the magnetic field generated by the magnet, respectively. The magnetic field $\boldsymbol{H}$ is calculated from the following relation; 


$$
\boldsymbol{H}=\frac{1}{\mu_{0} \chi_{\mathrm{m}}} \boldsymbol{M}
$$

where $\mu_{0}$ is the permeability in the free space.

In the magnetic field analysis, it is assumed that the magnetization $\boldsymbol{M}$ of the particles is parallel to the magnetic field $\boldsymbol{H}$ and angular momentum of the particles can be ignored. The magnetization of the magnetic particles is generally given by [14]

$$
\boldsymbol{M}=\phi M_{\mathrm{S}} L\left(\frac{\mu_{0} m|\boldsymbol{H}|}{k T}\right) \frac{\boldsymbol{H}}{|\boldsymbol{H}|}
$$

where $\phi$ is the concentration of the magnetic particles, $M_{\mathrm{S}}$ the saturation magnetization, $L$ the Langevin function, $k$ the Boltzmann constant, $T$ the absolute temperature, and $m=$ $V_{\mathrm{P}} M_{\mathrm{S}}$, where $V_{\mathrm{P}}$ is the volume of a magnetic particle. The magnetic susceptibility is dependent on the size of magnetic particle, but in this study, the magnetic field is so strong that the Langevin is equal to 1.0.

In order to deal with two or more kinds of magnetic particles, the magnetization $\boldsymbol{M}^{(e)}$ of the element $e$ is defined as

$$
\boldsymbol{M}^{(e)}=\frac{\boldsymbol{H}}{|\boldsymbol{H}|} \sum_{i=1}^{n} \phi_{i} M_{\mathrm{S} i}
$$

where $n$ is the number of kinds of magnetic particle. That is, the magnetization is represented as the summation of the magnetization of each particle, and the orientation of the magnetization is the same as that of the magnetic field. The concentration $\phi_{i}$ is given by solving the control volume equation.

The tetrahedral element is employed for solving (1) by the magnetic moment method.

\section{B. Control Volume Analysis}

To compute the concentration $\phi_{i}$ of $i$-th kind of the magnetic particle, the governing equation is as follows;

$$
\frac{\partial}{\partial}\left(\rho \phi_{i}\right)+\nabla \cdot\left(\rho v \phi_{i}\right)=\nabla \cdot\left(\Gamma \nabla \phi_{i}\right)+S
$$

where $\rho, v, \Gamma$, and $S$ are the fluid density, the fluid velocity, the scalar diffusion constant, and the scalar source term, respectively. Equation (5) is solved independently in every kind of the magnetic particle. The fluid velocity $v$ is given by the Navier-Stokes equation.

The ordinary finite element method with the common tetrahedral element is employed for solving (5) in this study.

\section{Navier-Stokes Analysis}

The governing equation of the fluid flow is

$$
\rho \frac{\partial \boldsymbol{v}}{\partial}+\rho \boldsymbol{v} \cdot \nabla \boldsymbol{v}=-\nabla p^{*}+\mu_{0}(\boldsymbol{M} \cdot \nabla) \boldsymbol{H}+\eta \nabla^{2} \boldsymbol{v}
$$

where $p^{*}$ and $\eta$ are the composite pressure and the coefficient of viscosity, respectively. The magnetization $\boldsymbol{M}$ and the magnetic field $\boldsymbol{H}$ are obtained from solving (1)-(4).

The Navier-Stokes equation is solved by the stabilized finite element method, that is the streamline upwind/Petrov-Galerkin and Pressure stabilizing/Petrov-Galerkin method, popular in the field fluid dynamics simulation [15].

\section{Magnetic Fluid Flow Analysis for Magnetic Separation}

For simulation of the magnetic separation, two or more kinds of magnetic particles have to be taken into account, however it is impossible to simulate it by any commercial software. Therefore, we have developed the simulation code to deal with two or more kinds of magnetic particles, solving (1)(6). Fig. 1 shows the flowchart of the developed simulation, the magnetic field, the Navier-Stokes, and the control volume analyses are done sequentially.

\section{FIG. 1 HERE}

The tetrahedral elements are commonly used in the magnetic field, the control volume, and the Navier-Stokes analysis. The magnetization $\boldsymbol{M}$ is constant in element, and the magnetic field $\boldsymbol{H}$, the magnetic fluid $\boldsymbol{v}$, and the concentration $\phi$ of magnetic particles are on node, as shown in Fig. 2.

\section{FIG. 2 HERE}

\section{Numerical Analysis for Magnetic Separation}

To investigate the validity of the developed simulation, the simulation results of the magnetic fluid flow with two kinds of the magnetic particles are compared with those of the magnetic fluid flow with one kind of the magnetic particle.

The flow channel of the magnetic chromatography used consists of nano-wires on upper and lower walls, as shown in Fig. 3. The height, width, and length of the flow channel are $0.17 \mathrm{~mm}, 2.0 \mathrm{~mm}$, and $2.5 \mathrm{~mm}$, respectively. The length is too short to separate the magnetic particles. However, it is shortened to compute the magnetic flow for simplicity. The superconducting magnet applies $2.0 \mathrm{~T}$ to the magnetic chromatography. The nano-wires of $0.2 \mathrm{~mm}$ width are alternately located on the upper and lower walls of the flow channel inside the magnetic column, which is made of $7 \%$ nickel and 93\% cobalt. The saturated magnetization of nanowire is $1.715 \mathrm{~T}$ in experiment when the over $0.1 \mathrm{~T}$ magnetic field is applied. The body of the magnetic column is made of nonmagnetic stainless steel.

The flowed magnetic particle is the fine $\mathrm{Fe}_{3} \mathrm{O}_{4}$ particle, its saturation magnetization is $0.9383 \mathrm{~T}$. The initial flow of the entrance of the flow channel is $5.0 \mathrm{~mm} / \mathrm{s}$. Table I shows the specifications of the magnetic fluid.

\section{TABLE 1 HERE}




\section{A. Simulation of One Kind of Magnetic Particle}

First of all, the magnetic fluid flow with two kinds of magnetic particle is individually simulated, their materials are same but their sizes are different, as shown in Table I. The magnetic particle with diameter of $30 \mathrm{~nm}$ and the diffusion coefficient of $10^{-2} \mathrm{~mm}^{2} / \mathrm{s}$ is called the magnetic fluid $A$, and the magnetic particle with $300 \mathrm{~nm}$ diameter and $10^{-3} \mathrm{~mm}^{2} / \mathrm{s}$ diffusion coefficient the magnetic particle $B$. Here, the diffusion coefficient is inversely proportional to the size of particle [14]. Fig. 4 shows the concentration of the magnetic particles and the flow in the case of the magnetic fluid $A$ on the vertical cross section at $t=0.2 \mathrm{~s}$. Figs. 5 and 6 show the enlarged view of the concentration of the magnetic particles and the flow of the magnetic fluid in the case of the magnetic fluids $A$ and $B$. Here, the front of the magnetic particles is also shown. The magnetic fluid meanders through the channel by the magnetic forces according to the magnetic gradients on vertical direction in the channel.

\section{B. Simulation of Two Kinds of Magnetic Particle}

The magnetic fluid flow simultaneously containing two kinds of magnetic particles are simulated by the developed simulation method. The materials of the magnetic particles are same but the sizes are different (see Table I). Fig. 7 shows the concentrations of the magnetic particles $A$ and $B$, and the flow of the magnetic fluid on the vertical cross section at $t=0.2 \mathrm{~s}$.

The magnetic fluid with particles $A$ and $B$ meanderingly flows also. However, the front of the magnetic particles $A$ and $B$ in Fig. 7 is obviously different from those of the separately simulated magnetic particles $A$ and $B$ in Figs. 5 and 6. The different magnetic force is generated since the composite magnetization is computed considering two kinds of magnetic particles at the same time, derived from (4).

The small differences of the front of the magnetic particles in Figs. 5, 6, and 7 are observed. However, the length of the simulated magnetic column is much shorter than that of the real developed magnetic column [6]. The difference of the velocity of magnetic particles becomes large in experiment.

\section{CONCLUSION}

For simulation of a magnetic separation system, it is desired to develop a magnetic fluid simulation tool taking into account magnetics and ferrohydrodynamics. Therefore, we have newly developed the simulation method to consider two or more kinds of the magnetic particles in magnetic fluid for magnetic separation. The proposed simulation method takes into account the magnetic field, the Navier-Stokes, and the control volume analyses in sequence. For consideration of two or more kinds of magnetic particles the composite magnetization is employed and the results of the proposed method are different from the results obtained by the separate simulation.

\section{ACKNOWLEDGMENT}

This work was supported in part by a Grant for Young Scientists (B) of the Ministry of Education, Culture, Sports, Science and Technology.

\section{REFERENCES}

[1] Y. Nakai, F. Mishima, Y. Akiyama, and S. Nishijima, "Development of magnetic separation system for powder separation," IEEE Trans. Appl. Supercond., vol. 20, no. 3, pp. 941-944, Jun. 2010.

[2] S. Hayashi, F. Mishima, Y. Akiyama, and S. Nishijima, "Development of high gradient magnetic separation system for a highly viscous fluid," IEEE Trans. Appl. Supercond., vol. 20, no. 3, pp. 945-948, Jun. 2010.

[3] H. Ueda, K. Agatsuma, S. Fuchino, T. Imura, M. Furuse, K. Kajikawa, A. Ishiyama, T. Koizumi, and S. Miyake, "Improvement of a highgradient magnetic separation system for trapping immunoglobulin in serum," IEEE Trans. Appl. Supercond., vol. 20, no. 3, pp. 949-952, Jun. 2010.

[4] T. H. Kim, D. W. Ha, J. M. Kwon, M. H. Sohn, S. K. Baik, S. S. Oh, R. K. Ko, H. S. Kim, Y. H. Kim, and S. K. Park, "Purification of the coolant for hot roller by superconducting magnetic separation," IEEE Trans. Appl. Supercond., vol. 20, no. 3, pp. 965-968, Jun. 2010.

[5] S. Noguchi and S. B. Kim, "Investigation on novel magnetic chromatography with ferromagnetic nano-wires for ion separation," IEEE Trans. Appl. Supercond., vol. 21, no. 3, pp. 2068-2071, Jun. 2011.

[6] S. B. Kim, C. Nakada, S. Murase, H. Okada, and T. Ohara, "Development of magnetic chromatography system for magnetic particle and ion separation with superconducting magnet," Physica C, vol. 463465, pp. 961-964, Jun. 2010.

[7] S. B. Kim, Y. Okimoto, S. Murase, S. Noguchi, and H. Okada, "Characteristics of magnetic separation for magnetic particle and ion by magnetic chromatography with novel magnetic column," IEEE Trans. Appl. Supercond., vol. 19, no. 3, pp. 2152-2156, Jun. 2009.

[8] S. Noguchi and S. B. Kim, "Development of a numerical simulation method for the magnetic separation of magnetic particles," IEEE Trans. Magn., vol. 47, no. 5, pp. 898-901, May 2011.

[9] G. Yoshikawa, K. Hirata, F. Miyasaka, and Y. Okaue, "Numerical analysis of transitional behavior of ferrofluid employing MPS method and FEM," IEEE Trans. Magn., vol. 47, no. 5, pp.1370-1373, May 2011.

[10] G. Yoshikawa, K. Hirata, and F. Miyasaka, "Numerical analysis of electromagnetic levitation of molten employing MPS method and FEM," IEEE Trans. Magn., vol. 47, no. 5, pp. 1394-1397, May 2011.

[11] K. H. Song, B. J. Park, and H. J. Choi, "Effect of magnetic nanoparticle additive on characteristics of magnetorheological fluid," IEEE Trans. Magn., vol. 45, no. 10, pp. 4045-4048, Oct. 2009.

[12] M. Pavel and A. Stancu, "Study of the optimum injection sites for a multiple metastases region in cancer therapy by using MFH," IEEE Trans. Magn., vol. 45, no. 10, pp. 4825-4828, Oct. 2009.

[13] Y. Takahashi, C. Matsumoto, and S. Wakao, "Large-scale and fast nonlinear magnetostatic field analysis by the magnetic moment method with adaptive cross approximation," IEEE Trans. Magn., vol.43, no. 4, pp. 1277-1280, Apr. 2007

[14] R. E. Rosensweig, Ferrohydrodynamics. New York: Dover, 1997.

[15] J. H. Ferziger and M. Peric, Computational Methods for Fluid Dynamics. Berlin, Germany: Springer-Verlag, 1996.

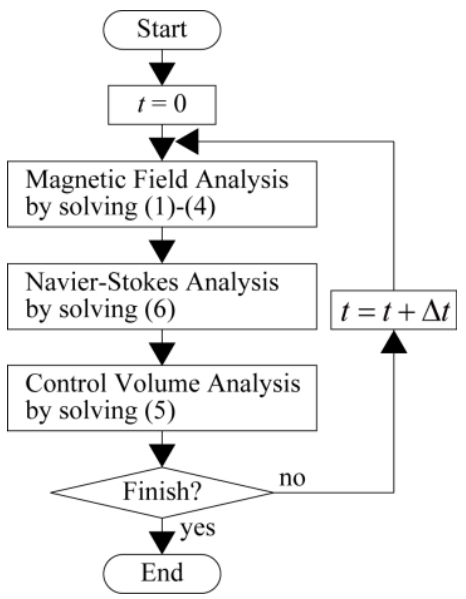

Fig. 1. Flowchart of the developed ferrohydrodynamics and magnetic field simulation. The magnetic field, the Navier-stokes, and the control volume analyses are done sequentially. 


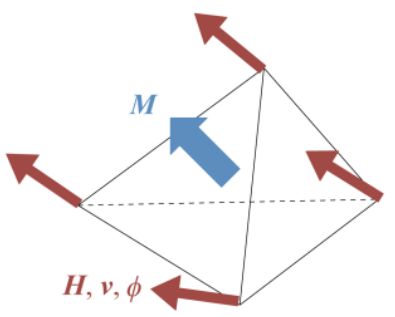

Fig. 2. Physical quantity computed in the developed simulation. The tetrahedral elements are commonly used in the magnetic field, the control volume, and the Navier-Stokes analysis. The magnetization $\boldsymbol{M}$ is constant in element. The magnetic field $\boldsymbol{H}$, the fluid velocity $\boldsymbol{v}$, and the concentration $\phi$ of the magnetic particles are on nodes.

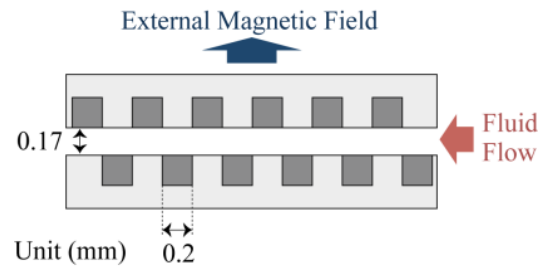

Fig. 3. Schematic view of the flow Channel of the developed magnetic column.

TABLE I

PROPERTIES OF MAGNETIC FLUID

\begin{tabular}{ll}
\hline Magnetic particle & $\mathrm{Fe}_{3} \mathrm{O}_{4}$ \\
Saturated magnetization $(\mathrm{T})$ & 0.9383 \\
Initial velocity $(\mathrm{mm} / \mathrm{s})$ & 5.0 \\
Average diameter of particle $(\mathrm{nm})$ & 30,300 \\
Diffusion coefficient $\left(\mathrm{mm}^{2} / \mathrm{s}\right)$ & $10^{-2}, 10^{-3}$ \\
Viscosity coefficient $(\mathrm{g} / \mathrm{ms})$ & 1.0 \\
\hline
\end{tabular}

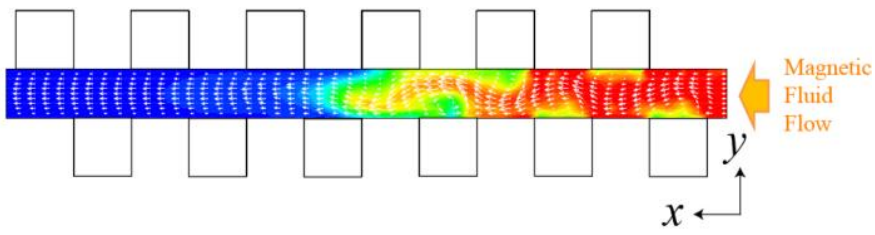

(a)

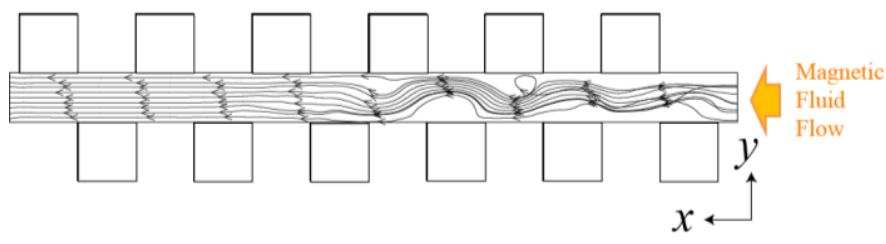

(b)

Fig. 4 Separately simulated flow of the magnetic fluid $A$ at $t=0.2 \mathrm{~s}$. (a) concentration of the magnetic particles, and (b) flow of the magnetic fluid. The meandering flow is observed.

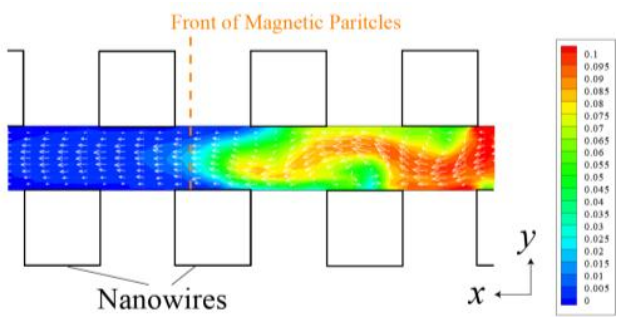

(a)

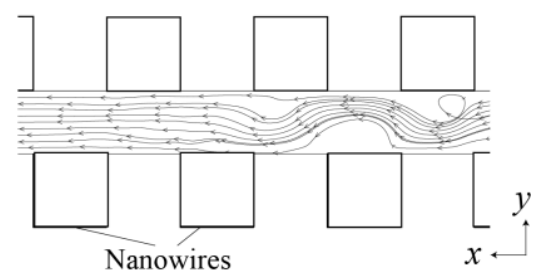

(b)

Fig. 5 Enlargement view of the separately simulated flow of the magnetic fluid $A$ at $t=0.2 \mathrm{~s}$. (a) concentration of the magnetic particles, and (b) flow of the magnetic fluid.

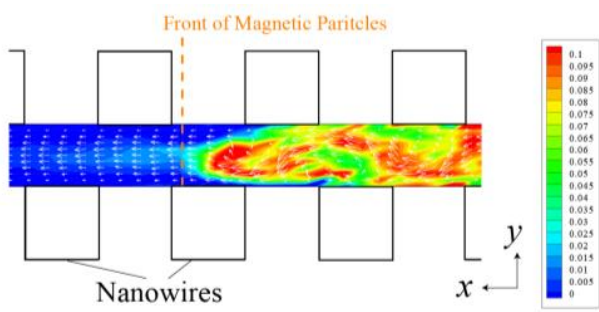

(a)

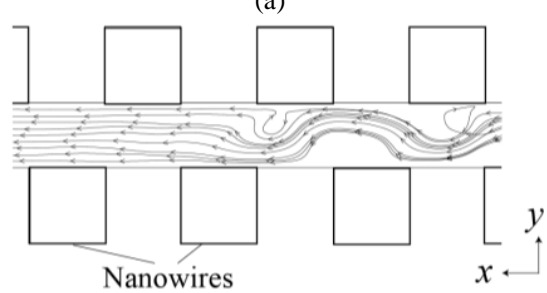

(b)

Fig. 6 Enlargement view of the separately simulated flow of the magnetic fluid $B$ at $t=0.2 \mathrm{~s}$. (a) concentration of the magnetic particles, and (b) flow of the magnetic fluid.

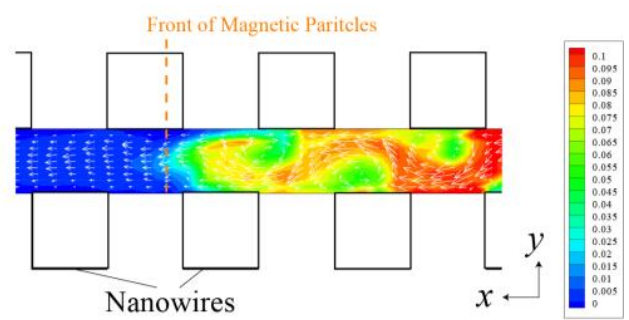

(a)

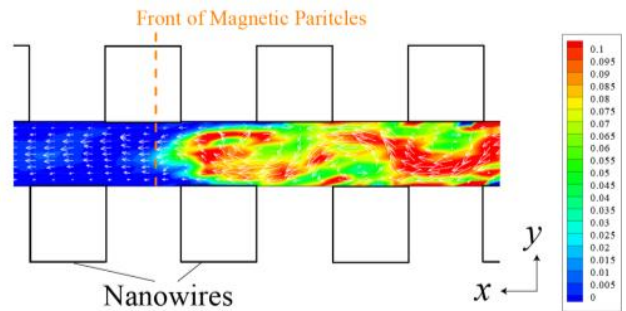

(b)

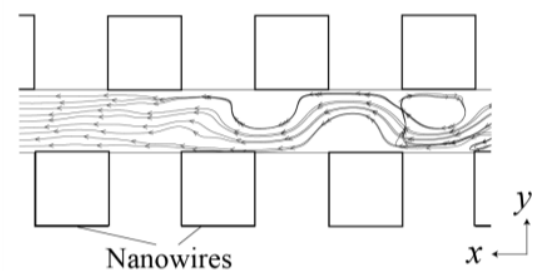

(c)

Fig. 7 Simultaneous simulated flow of the magnetic fluids $A$ and $B$ at $t=0.2 \mathrm{~s}$. (a) concentration of the magnetic particles $A$, (b) concentration of the magnetic particles $B$, and (c) flow of the magnetic fluid. 